Instituto Internacional de Investigación y Desarrollo Tecnológico Educativo INDTEC, C.A.

DOI: https://doi.org/10.29394/Scientific.issn.2542-2987.2020.5.17.14.267-279

OAI-PMH: http://www.indteca.com/ojs/index.php/Revista Scientific/oai

Ensayo Original / Original Essay

\title{
Uso del Smartphone por jóvenes estudiantes de secundaria: Una mirada crítica
}

Autora: Jerardin del Carmen Ríos Anciani Universidad Nacional Experimental "Rafael María Baralt", UNERMB jerardinrios@gmail.com Zulia, Venezuela https://orcid.org/0000-0003-4926-6435

\section{Resumen}

El presente ensayo tiene como objetivo analizar desde una mirada crítica los inconvenientes o efectos asociados al uso y abuso de los teléfonos inteligentes por pate de los jóvenes estudiantes de secundaria. Sin duda, los Smartphone, se han convertido en un objeto de gran valor para los jóvenes, quienes son los más susceptibles de ser influenciados por su uso y atractivo. Lo definen como una extensión de su cuerpo, una parte fundamental de sus vidas que los mantiene ocupados gran parte del día y de la noche, pasando largas horas conectados con el Aparato, por tanto, su uso excesivo, genera una serie de inconvenientes, en lo familiar, social, académico y su salud.

Palabras clave: teléfono móvil; joven; estudiante.

Cómo citar este ensayo:

Ríos, J. (2020). Uso del Smartphone por jóvenes estudiantes de secundaria: Una mirada crítica. Revista Scientific, 5(17), 267-279, e-ISSN: 2542-2987. Recuperado de: https://doi.org/10.29394/Scientific.issn.2542-2987.2020.5.17.14.267-279

Fecha de Recepción: 09-03-2020
Fecha de Aceptación: 26-06-2020
Fecha de Publicación: 05-08-2020 
Instituto Internacional de Investigación y Desarrollo Tecnológico Educativo INDTEC, C.A.

DOI: https://doi.org/10.29394/Scientific.issn.2542-2987.2020.5.17.14.267-279

OAI-PMH: http://www.indteca.com/ojs/index.php/Revista_Scientific/oai

Ensayo Original / Original Essay

\title{
Smartphone use by young high school students: A critical look
}

\begin{abstract}
The objective of this essay is to analyze from a critical perspective the disadvantages or effects associated with the use and abuse of smartphones by pate of young high school students. Without a doubt, the Smartphone has become an object of great value for young people, who are the most likely to be influenced by its use and attractiveness. They define it as an extension of their body, a fundamental part of their lives that keeps them busy much of the day and night, spending long hours connected to the Device, therefore, its excessive use generates a series of inconveniences, in family, social, academic and health.
\end{abstract}

Keywords: mobile phone; youth; student.

How to cite this essay:

Ríos, J. (2020). Smartphone use by young high school students: A critical look. Revista Scientific, 5(17), 267-279, e-ISSN: 2542-2987. Recovered from: https://doi.org/10.29394/Scientific.issn.2542$\underline{2987.2020 .5 .17 .14 .267-279}$

Date Received:

09-03-2020
Date Acceptance:

26-06-2020
Date Publication:

05-08-2020 


\section{Introducción}

El hombre desde los inicios de la humanidad ha sentido la necesidad de comunicarse y relacionarse con los otros, bien sea a través del lenguaje hablado, la lengua escrita, por medio de gestos o expresiones corporales. La invención de diversos aparatos y herramientas tecnológicas como: la imprenta, la televisión, computadores, la telefonía fija y móvil, le ha permitido ampliar, y mejorar los procesos comunicativos entre las personas.

Ahora bien, desde la aparición del primer teléfono celular el DynaTAC, 8000X, de la industria de telecomunicaciones Motorola, en el año 1983, cambió drásticamente la manera de comunicarse lógicamente, para ese tiempo era inimaginable la idea de entablar conversaciones con otras personas a distancia. Hoy día, han pasado ya 36 años, la evolución tecnológica ha revolucionado por completo el estilo de vida de las personas, los celulares y en especial los teléfonos inteligentes, (Smartphone) entretienen, informan, conectan, y permiten dejar huella de todos los momentos que vive un individuo en su acontecer diario.

Es así como los Smartphone, han causado un gran impacto en la sociedad Venezolana llegando a cambiar sus costumbres y estilo de vida, .principalmente en los jóvenes, que al entrar la etapa del Bachillerato experimentan una serie de cambios en busca de su identidad y personalidad, por tanto, son más susceptibles de ser influenciados por los nuevos aparatos e innovaciones tecnológicas principalmente debido las características que ofrecen estos productos de alta gama como las ventajas que representa estar permanentemente conectado a internet, lo cual permite el acceso a las diferentes redes sociales como (Twitter, Facebook, Instagram), y capacidad para instalar todo tipo de juegos y aplicaciones (Apps), las más populares entre los jóvenes: son el WhatsApp, el Facebook y el Instagram).

Con relación a lo antes expuesto, el Smartphone se ha convertido en un anhelo por muchos jóvenes, una necesidad para ellos, un objeto preciado, 
Instituto Internacional de Investigación y Desarrollo Tecnológico Educativo INDTEC, C.A.

DOI: https://doi.org/10.29394/Scientific.issn.2542-2987.2020.5.17.14.267-279

OAI-PMH: http://www.indteca.com/ojs/index.php/Revista_Scientific/oai

Ensayo Original / Original Essay

a través del cual pueden ser ellos mismos. Los jóvenes quieren ser escuchados, quieren que los demás los vean sepan lo que están haciendo, quienes son, como son, y decir lo que son, esto lo logran a través de su Smartphone, es así como el contacto personal se pierde en la virtualidad.

Lo anterior, coincide con lo expuesto por Peñalver (2016), quien sostiene que:

Estos jóvenes quieren ser escuchados, quieren aportar valor con sus opiniones, quieren ganar, competir, sobresalir, siendo héroes y sobre todo cumplir una misión. Usan el internet como un medio de socialización donde se crean y recrean relaciones, construyendo una relación de amistad de 24 horas al día, en otras palabras, son los protagonistas, guionistas, productores y directores de su historia (pág. 192).

Por su parte, las organizaciones educativas se jactan del papel protagónico que cumple el estudiante, sin embargo, están dejando de lado el estado emocional, intereses, percepciones y hábitos de los estudiantes quienes son la razón del hecho educativo.

Ante esa realidad, los docentes, y miembros de las instituciones que ofrecen educación a nivel medio diversificado, deben enfocar su atención en conocer a los estudiantes sus intereses y particularidades, asumiendo una postura flexible, humanista y estar abierto a los cambios, para saber llevar dentro de un entorno de reflexión y comprensión la formación de sus estudiantes.

En la actualidad es notable el uso del Smartphone dentro y fuera de las aulas de clase, como una práctica común entre los jóvenes cuando están en horas de receso. Más aún, se han presentado algunos incidentes dentro de las aulas de clase, debido a que los estudiantes de manera deliberada utilizan el Smartphone sin consentimiento de los profesores, actuando entonces como un agente distractor dentro de procesos formativos.

Dentro de ese contexto, es común observar en los pasillos de liceos 
grupos de estudiantes aparentemente compartiendo entre compañeros, demostrándose entre sí poco contacto visual e interacción persona a persona, pues la interacción que se nota es la del individuo con su Smartphone, y el resto del entorno se pierde en la virtualidad. Pareciese que este aparato formara parte de ellos, una extensión de ellos mismos, un objeto de vital importancia.

Ante esto, Malo (2006): manifiesta que "una consecuencia inmediata de este consumismo: la exclusión" (pág. 111); se basa en las personas que no tienen un teléfono inteligente, es posible estén destituidos del circulo digital de amigos que envuelve este siglo XXI.

En este sentido, las consecuencias de esto se reflejarían en la ausencia de integración de todos los involucrados en el proceso educativo; manteniendo por tanto, una desconexión constante y permanente con la realidad y el entorno, lo cual estaría interfiriendo de manera directa con el establecimiento no solo de relaciones interpersonales poco saludables, sino de una débil contribución en la construcción del aprendizaje integral. De acuerdo con García, (2013), citado por Capilla y Cubo (2017a): puede surgir en los usuarios una "pérdida de control, la búsqueda desesperada por obtener refuerzos rápidos y sentimientos de malestar si no usa las tecnologías suficientemente" (pág. 177).

Asimismo, destacan Silva y Martínez (2017a): que "a nivel pedagógico, los dispositivos se constituyen en herramientas de interacción, pero se corre el riesgo de implementar fuentes de distracción y pérdida de la atención de los estudiantes" (pág. 12). Sin lugar a duda, el tiempo que le dedican los estudiantes a este aparato pudiera afectar diferentes aspectos de su vida, incluyendo su desempeño académico.

Habiendo evidenciado la dimensión del fenómeno social tecnológico, el objetivo del presente ensayo es analizar los inconvenientes o efectos asociados al uso y abuso del smartphone por parte de los jóvenes estudiantes 
de educación secundaria.

\section{Desarrollo}

\subsection{Teléfonos Inteligentes}

Es un teléfono celular (móvil) que ofrece a los usuarios funcionalidades similares a de un computador destacándose por su capacidad de conexión a internet a través de paquetes de datos y vía WiFi. Este tipo de teléfonos de última generación cuentan con todas las funciones básicas de un celular común, (llamadas telefónicas, envío de mensajes de texto, reloj, alarmas, calendarios etc.) pero con características avanzadas (pantalla táctil, tamaño atractivo, capacidad multimedia, amplia memoria, cámara de alta resolución, grabador, reproductor de audios MP3 y videos MP4, conexión a internet, 3G y 4G, aplicaciones variadas, hasta visualizar documentos de trabajo creados en formato PDF u otros formatos digitales, etc.).

De acuerdo con González (2007), en lo que respecta al teléfono móvil:

El celular es visto como un medio de comunicación, integrándose dentro del grupo constituido por las nuevas tecnologías y medios de comunicación (por ejemplo: internet, canales de chat, blogs, fotologs), nutriéndose de los criterios de comunicación impuestos (tanto por los nuevos medios como por los viejos) y estableciendo otros nuevos [...] (pág. 81).

Es decir, haciendo referencia a los Smartphone o teléfonos inteligentes, se definen como una minicomputadora portátil. Entre los fabricantes de esta clase de dispositivos están: Motorola, Sony, Samsung, Nokia, LG, BlackBerry Alcatel, y Huawei. Básicamente, el teléfono celular, constituye una de las herramientas tecnológicas con mayor demanda de compra, y los usuarios más asiduos de ella lo constituyen principalmente los adolescentes quienes lo consideran como un elemento fundamental en sus vidas, por ser un dispositivo que los mantiene conectados constantemente con sus familiares o amigos, integrar grupos, investigar, y compartir su propia vida a través de la internet. 
Una característica esencial del Smartphone es su conectividad a internet, y la rapidez con la que accede a los datos.

\subsection{Uso de Teléfonos inteligentes por los jóvenes}

Los teléfonos inteligentes conocidos también como Smartphone son posiblemente el elemento más común en la vida diaria, casi tanto como la vestimenta, ya que, en la actualidad, salir de casa sin el celular es inconcebible, sería como salir desnudo, o sentirse vacío y aburrido durante todo el día. Esta analogía refleja una realidad de la que todas las personas forman parte, pero sin duda alguna los jóvenes son los más vulnerables de ser influenciados por el uso de esta tecnología y dramatiza más en aquellos que utilizan de manera exagerada este dispositivo hasta el punto de convertirse en una dependencia al teléfono inteligente.

Actualmente es común observar a los jóvenes que llevan a todas partes sus teléfonos inteligentes con la finalidad de estar permanentemente en interacción con las otras personas a través de redes sociales o estar realizando otro tipo de actividad de entretenimiento u ocio, como escuchar música, ver videos en YouTube o enviando mensajes de texto. Hoy día es normal tomar selfis en diferentes momentos del día y subirla a las redes o compartirlas sociales. Los jóvenes perciben el móvil como un objeto que refleja su identidad.

Asumo que este dispositivo de alta tecnología, es utilizado por muchos jóvenes para darse a conocer, mostrarse de una manera diferente a como lo perciben en la realidad. A través del uso del Smartphone, pueden permanentemente estar conectados con otras personas y mostrarse tal cual ellos quieren que los vean, mediante la utilización de Emoji, Memes, Músicas, Videos, Blogs y Facebook.

Es importante destacar que además de la cantidad de jóvenes que poseen teléfonos inteligentes, el número de horas que pasan conectados a la 
red supera las tres (3) horas, inclusive en el horario nocturno pasan mucho tiempo durante la noche hablando por chats, o en la redes como Facebook o WhatsApp, asimismo la edad en la que reciben estos jóvenes reciben este aparato que en primera instancia es entregado bajo su responsabilidad con fines de estar en comunicación con sus padres principalmente, es cuando entran a la etapa de educación media. Esto concuerdan con lo expresado por Martínez, Enciso y González (2015), citados por Silva y Martínez (2017b), con respecto a la edad de uso de los teléfonos móviles:

Los niños y niñas les piden a sus padres que les compren el aparato cada vez a más temprana edad, oscila entre los 11 a 12 años, pero se han dado casos de niños y niñas de 6 a 7 años con móvil en la escuela (pág. 13).

Comúnmente los jóvenes utilizan el teléfono inteligente dentro de las categorías siguientes, o bien con los siguientes fines: comunicación, entretenimiento, satisfacción personal y vigilancia.

\subsection{Inconvenientes u efectos asociados al uso excesivo de Teléfonos Inteligentes}

Los teléfonos inteligentes han llegado a cambiar el estilo de vida de las personas, sus formas de comunicarse, asociarse, aprender y desenvolverse en la sociedad están siendo afectados hasta tal punto que algunos jóvenes definen este aparato como una extensión de su propio cuerpo y expresiones como: Sin el celular no puedo vivir, el celular es mi vida, No sé qué haría sin mi celular, son las expresiones más comunes de los jóvenes cuando se les pregunta con relación a su teléfono.

Dentro de este contexto, enfatiza Organista-Sandoval, McAnally-Salas y Lavigne (2013): que el teléfono inteligente "[...] posee capacidades técnicas interesantes que han propiciado su aceptación [...]" (pág. 8). En este sentido, cabe destacar que, desde la aparición de los teléfonos inteligentes muchas 
actividades se han simplificado como el acceso a información inmediata a través de la conexión a internet, comunicación a distancia y mensajería instantánea, también ha creado algunos inconvenientes que pudieran denotar en un tipo de adicción a la tecnología cuyos efectos estarían afectando su vida a nivel social, personal y académico.

En consecuencia, el uso de los Smartphone llega a volverse un problema cuando los usuarios se vuelven esclavos de él, básicamente no pueden separase del aparato, y el grado de ansiedad aumenta notoriamente, dándole un mal uso a la tecnología. Algunos de los síntomas de abuso del teléfono inteligente se ponen en evidencia cuando la persona comienza a desarrollar indicios de dependencia como aislamiento, bien sea que este solo o acompañado, distracción, y atención focalizada en el uso del aparato tecnológico como Smartphone o tablets.

Lo planteado anteriormente, Silva y Martínez (2017c), sostienen que:

Algunas nuevas formas de comportamiento se relacionan con la dependencia, la abstracción de la realidad, un excesivo conectivismo, sedentarismo e incluso a suplir necesidades de asociación, autorrealización y autoestima (pág. 13).

Por su parte, Capilla y Cubo (2017b), afirman que: "las enfermedades tecnológicas aumentan por el uso excesivo de la misma [...]" (pág. 178). Dicho autor comenta que el abuso de estas tecnologías puede provocar en los usuarios falta de memoria operativa, problemas de la vista y de audición, entre otros. Además, pueden presentarse otros problemas como ansiedad, síndrome del túnel carpiano, vista cansada, dolor de cabeza, dolor de espalda, lesiones en el pulgar, etc.

\section{Conclusiones}

Ser propietario de un teléfono celular, muy específicamente un teléfono inteligente, o Smartphone, sea convertido en los últimos años en una 
necesidad, no solo por las múltiples funcionalidades que tiene como: envío de mensajes de texto o multimedia, llamadas, cámara fotográfica, video, calculadora, reproductor de música, ver la hora, principalmente la posibilidad de estar conectados a través de internet, lo que permite además, investigar, realizar todo tipo de operaciones académicas, bancarias y estar en comunicación con muchas personas a distancia mediante el uso de las redes sociales como: Instagram, Twitter, y las más utilizadas por los jóvenes: WhatsApp y Facebook.

Por consiguiente, los jóvenes ante el sinnúmero de funcionalidades que les brinda el Smartphone son susceptibles de ser influenciados por tan llamativo aparato, de tal manera que pasan horas conectados en internet a través del Smartphone, de día y de noche. Los jóvenes suelen estar hasta altas horas de la noche en las redes sociales chateando con amigos, compartiendo información o actualizando estados, al igual que WhatsApp.

Indudablemente la forma de comunicarse ha cambiado, dando prioridad a las comunicaciones vía internet, que a la comunicación persona a persona. Por un lado, las relaciones interpersonales se ven afectadas, ya que el usuario pierde el contacto real con la persona y este es reemplazado por el contacto mediado a través del Smartphone. Asimismo, muchos de los jóvenes que antes solían realizar todo tipo de actividades al aire libre, salir con amigos, ir a clubes, al parque, conversar en reúnes familiares y de amigos, ahora esto se ve afectado por el uso del Smartphone.

En cuanto a lo anterior, los efectos causados por el uso excesivo del Smartphone, en ocasiones son negativos. No obstante, el Smartphone también puede ser utilizado por los jóvenes estudiantes como un recurso que les permite investigar, comunicarse con sus seres queridos y familiares, profesores, compañeros de clase, utilizar herramientas como: calculadora, cámara, reloj, entre otras que facilitan su trabajo estudiantil. No obstante, es importante que tanto la familia como las instituciones educativas establezcan 
normas para el uso responsable de este dispositivo y sacarles el mejor provecho.

Si bien es cierto que el uso de los Smartphone no está influyendo en el desempeño de los estudiantes, si afecta el tiempo que le dedican a lo académico, familiar y social, en el ámbito personal respectivamente. Este es un llamado a la reflexión de todos los actores educativos para que tomen en cuenta esta realidad que pudiera en cualquier momento salirse de las manos y causas mayores inconvenientes a las personas.

\section{Referencias}

Capilla, E., \& Cubo, S. (2017a,b). Phubbing. conectados a la red y desconectados de la realidad. un análisis en relación al bienestar psicológico. Pixel-Bit: Revista de Medios y Educación, (50), 173-185, e-ISSN: 1133-8482 Recuperado de:

https://www.redalyc.org/articulo.oa?id=36849882012

González, L. (2007). Representación social del teléfono celular. Trabajo final. Argentina: Universidad Empresarial Siglo 21. Recuperado de: https://repositorio.uesiglo21.edu.ar/handle/ues21/12283

Malo, S. (2006). Impacto del teléfono móvil en la vida de los adolescentes entre 12 y 16 años. Comunicar, (27), 105-112, e-ISSN: 1134-3478. Recuperado de:

https://www.redalyc.org/articulo.oa?id=15802716

Organista-Sandoval, S., McAnally-Salas, L., \& Lavigne, G. (2013). El teléfono inteligente (smartphone) como herramienta pedagógica. Apertura, 5(1), 6-19, e-ISSN: 1665-6180. Recuperado de:

http://www.redalyc.org/articulo.oa?id=68830443002

Peñalver, M. (2016). Uso del celular inteligente (Smartphone) dentro del contexto educativo formal: Una visión sociocultural. CIEG, (26), 185-201, e-ISSN: 2244-8330. Barquisimeto, Venezuela: Centro de 
Instituto Internacional de Investigación y Desarrollo Tecnológico Educativo INDTEC, C.A.

DOI: https://doi.org/10.29394/Scientific.issn.2542-2987.2020.5.17.14.267-279

OAI-PMH: http://www.indteca.com/ojs/index.php/Revista_Scientific/oai

Ensayo Original / Original Essay

Investigación y Estudios Gerenciales (CIEG).

Silva, A., \& Martínez, D. (2017a,b,c). Influencia del Smartphone en los procesos de aprendizaje y enseñanza. Suma de Negocios, 4(17), 11-

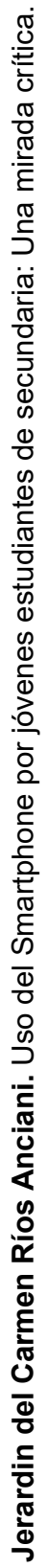

18, e-ISSN: 2215-910X. Recuperado de:

http://dx.doi.org/10.1016/j.sumneg.2017.01.001 


\section{Jerardin del Carmen Ríos Anciani}

e-mail: jerardinrios@gmail.com

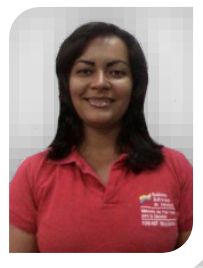

Nacida en Cabimas estado Zulia, Venezuela, el 4 de febrero del año 1985. Docente investigadora; tengo estudios de Pregrado con Titulo de Licenciada en Educación Integral por la Universidad Nacional Experimental "Rafael María Baralt" (UNERMB, 2007); Magister Scientiarum en Administración de la Educación por la Universidad Nacional Experimental "Rafael María Baralt" (UNERMB, 2005); cuento con otros estudios como Técnico Superior Universitario en Informática por el Programa Nacional de Formación PNF Misión Sucre (2019); Experiencia laboral como Profesora Asesora en la Misión Sucre PNF Educación (2007-2010); Docente de Centro Bolivariano de Informática y Telemática (CBIT), en la Escuela Básica Nacional Santa Rita (2008-2018); y Docente de Recursos para el Aprendizaje, desde el año 2018 hasta la actualidad, en la Unidad Educativa "Nuestra Señora del Rocío".

El contenido de este manuscrito se difunde bajo una Licencia de Creative Commons ReconocimientoNoComercial-Compartirlgual 4.0 Internacional 